\title{
Adrenal Insufficiency by Adrenoleukodystrophy
}

\author{
Elda Cristinne Mattos Botelho1, Eduardo Romero Sampaio Botelho1, \\ Luciana Corrêa de Souza Rodrigues', Tatiana Prado Wanderley¹, Fernanda Navarini Queiroz, \\ Pollyana Cirino Gomes Borges', Sabrina Arrais Rolim Aragão Ximenes Cruz ${ }^{1}$, \\ Janete Pacheco da Silva1, Larissa Bianca Cunha de Sá ${ }^{1}$, Alberto Krayyem Arbex ${ }^{1,2}$
}

${ }^{1}$ IPEMED Medical School, AFYA Educational, Brasília, Brazil

${ }^{2}$ Medical Clinic in Endocrinology and Diabetology, Schleswig-Flensburg, Germany

Email: elda_cris@hotmail.com

How to cite this paper: Botelho, E.C.M., Botelho, E.R.S., de Souza Rodrigues, L.C., Wanderley, T.P., Queiroz, F.N., Borges, P.C.G., Cruz, S.A.R.A.X., da Silva, J.P., de Sá, L.B.C. and Arbex, A.K. (2020) Adrenal Insufficiency by Adrenoleukodystrophy. Health, 12, 1-13.

https://doi.org/10.4236/health.2020.121001

Received: August 30, 2019

Accepted: January 3, 2020

Published: January 6, 2020

Copyright $\odot 2020$ by author(s) and Scientific Research Publishing Inc. This work is licensed under the Creative Commons Attribution International License (CC BY 4.0).

http://creativecommons.org/licenses/by/4.0/

\begin{abstract}
The X-linked adrenoleukodystrophy (ALD) is a severe neurodegenerative disorder due to mutations in the $\mathrm{ABCD} 1$ gene. Objective: To report a case of a 19-year-old man with adrenal insufficiency due to adrenoleukodystrophy. Method: Case report and literature review. Result: A previously healthy 19-year-old male patient was admitted to the emergency room with nausea and vomiting for 5 days, who progressed to abdominal pain, severe asthenia, and fever $\left(38.5^{\circ} \mathrm{C}\right)$. He referred progressive darkening of the skin, oral mucosa, tongue and nail bed of the hands and feet, observed in the last 6 years. Emergency laboratory evaluation showed severe hyponatremia and hyperkalemia, which, together with decreased plasma cortisol, directed the investigation to causes of adrenal insufficiency. High ACTH (Adrenocorticotropic hormone) and very long chain fatty acid levels closed the diagnosis. Discussion: ALD is characterized by progressive demyelination in the central and peripheral nervous system and adrenal insufficiency consequence to the accumulation of very long chain fatty acids (VLCFA) in the adrenal. The overall incidence of ALD is 1:17,000. Adrenal insufficiency may be the first symptom of ALD in boys and adults. The diagnosis is based on the measurement of VLCFA plasma levels. Allogeneic bone marrow transplantation is the only treatment that provides a permanent cure when the procedure is performed at an early stage of brain demyelination, i.e. when patients are asymptomatic, although brain magnetic resonance imaging (MRI) is abnormal. Treatment of Addison's disease is obligatory, but does not change the course of neurological symptoms.
\end{abstract}

\section{Keywords}

Adrenoleukodystrophy, Adrenomyeloneuropathy, Adrenal Insufficiency, 


\section{Introduction}

Adrenal insufficiency is the deficiency of hormones produced by the adrenal gland cortex. It has a prevalence of 5 out of 10,000 individuals in the general population [1]. The most common cause is autoimmune disease, accounting for $70 \%$ to $90 \%$. The rest consists of infectious, neoplastic, hemorrhagic, drug, and hereditary causes such as adrenoleukodystrophy (ALD) or adrenomyeloneuropathy (AMN) [2].

Adrenal insufficiency in ALD occurs by mutations in the ABCD1 gene that leads to the accumulation of lipid inclusions represented by very long chain fatty acids (VLCFA) in adrenal tissue. This excess of VLCFA causes altered adrenal cortex response to ACTH stimulation, increased adrenal cellular apoptosis and reduced cholesterol available for cortisol and androgen steroidogenesis. In young men, the presence of adrenal insufficiency should raise the possibility of adrenoleukodystrophy or adrenomyeloneuropathy as the cause of the disease. The diagnosis of ALD can be made biochemically before the appearance of histopathological changes and consequent hormonal deficiency [3].

Approximately two thirds of male patients have evident or subclinical adrenal insufficiency and a smaller proportion have associated gonadal dysfunction. Neurological abnormalities may appear before adrenal insufficiency, but up to $60 \%$ of patients have no neurological symptoms at the time of diagnosis of adrenal insufficiency [4].

The diagnosis is based on the measurement of VLCFA plasma levels, which allow the identification of $100 \%$ of affected men and $80 \%-95 \%$ of heterozygous women. Because of the false negative results, it is obligatory to look for a mutation in the $\mathrm{ABCD} 1$ gene in all women at risk of being heterozygous for ALD [5]. Allogeneic bone marrow transplantation is the only treatment that provides a permanent cure for the disease. This procedure is performed at an early stage of brain demyelination, that is, when patients are asymptomatic, although brain MRI is abnormal. Treatment of Addison's disease is obligatory and life-saving, but does not change the course of neurological symptoms. Dietary therapy failed to halt neurological progression in cerebral ALD and AMN [6].

\section{Case Description}

DGR, male, 19 years old, previously healthy: An informed consent was obtained from the patient to report the case in a medical research media. He was admitted to the emergency room with nausea and vomiting for 5 days, which progressed to abdominal pain, severe asthenia and fever. He referred progressive darkening of the skin, oral mucosa, tongue and nail bed of the hands and feet, observed in the last 6 years. 
On physical examination, the patient was dehydrated, tachycardic, with postural hypotension and moderate abdominal pain on palpation. Emergency laboratory evaluation showed severe hyponatremia $(\mathrm{Na} 116 \mathrm{mEq} / \mathrm{L}$, Normal Range: 135 - 145) and hyperkalemia (K $5.3 \mathrm{mEq} / \mathrm{l}$, NR: 3.8 - 5.2), which together with decreased plasma cortisol ( $2.3 \mathrm{ng} / \mathrm{dl}$, NR: 2 - 20) directed the investigation to causes of failure adrenal gland, of probable primary etiology, due to the finding of cutaneous and mucosal hyperpigmentation. Figures 1(a)-(c) show the most important features of the physical examination.

Treatment with venous hydrocortisone was initiated, which at discharge was replaced by oral glucocorticoid (Prednisone $5 \mathrm{mg} /$ day), combined with fluodrocortisone $0.1 \mathrm{mg}$, remaining stable clinically and laboratory.

During ambulatory follow-up, ACTH was extremely high $(>1250 \mathrm{pg} / \mathrm{ml})$, which confirmed the diagnosis of primary adrenal insufficiency (Addison's disease). In the etiological investigation, the diagnoses of tuberculosis, viral infections, autoimmune adrenalitis (anti-adrenal negative $-21 \mathrm{OH}$ ) were discarded and a very long chain fatty acid profile (peroxisomal diseases) compatible with the diagnosis of X-linked adrenoleukodystrophy was evidenced. Table 1 shows the serum values before and after the clinical approach.

\section{Literature Review}

\subsection{Adrenoleukodystrophy}

Adrenoleukodystrophy (ALD) is a neurodegenerative genetic disease included in the group of leukodystrophies [7]. It is considered a peroxisomal demyelinating disorder and it is caused by mutations in the ABCD1 gene that maps the Xq28 locus, which leads to accumulation of very long chain fatty acids in plasma and tissues [2]. No correlation was demonstrated between phenotype and the ABCD1 mutation [8].

The classic or infantile form is the most severe and most common although there are forms such as neonatal and adult forms. The infantile form was noted in the film "Lorenzo's Oil” (Lorenzo's Oil, USA, 1992), directed by George Miller, which the disease was excellently exemplified. This film emphasized not only

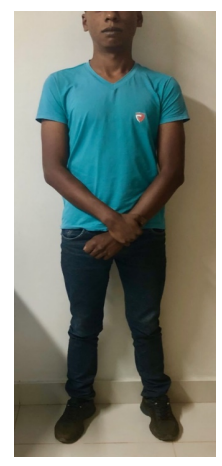

(a)

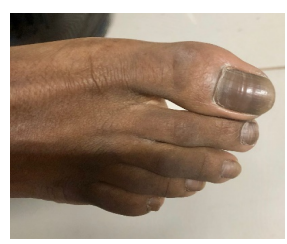

(b)

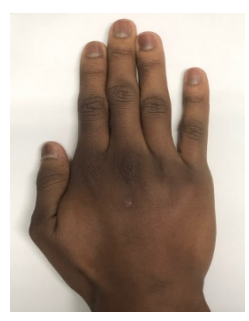

(c)

Figure 1. Clinical features of the case. 
Table 1. Serum values before and after treatment. Very long chain fatty acid profile. Gas chromatography method. Material: plasma.

\begin{tabular}{ccccc}
\hline & $07 / 22 / 2016$ & Reference value & $01 / 17 / 2018$ & Reference value \\
\hline Hexacosanoic acid 26:0 & 2.820 micromol/L & 0.22 to 0.88 micromol/L & 0.95 micromol/L & 0.22 to $0.88 \mathrm{micromol} / \mathrm{L}$ \\
Docosanoic acid 22:0 & $38.50 \mathrm{micromol} / \mathrm{L}$ & 51.10 to $113.40 \mathrm{micromol} / \mathrm{L}$ & $23.75 \mathrm{micromol} / \mathrm{L}$ & 51.10 to $113.40 \mathrm{micromol} / \mathrm{L}$ \\
Tetracosanoic acid 24:0 & $74.3 \mathrm{micromol} / \mathrm{L}$ & 44.30 to $92.40 \mathrm{micromol} / \mathrm{L}$ & $39.18 \mathrm{micromol} / \mathrm{L}$ & 44.30 to $92.40 \mathrm{micromol} / \mathrm{L}$ \\
$24: 0 / 22: 0$ & 1.930 & 0.55 to 0.89 & 1.65 & $<1.36$ \\
$26: 0 / 22: 0$ & 0.073 & 0.004 to 0.021 & 0.040 & $<0.022$ \\
\hline
\end{tabular}

the patient's suffering but also the family repercussion of the perception of his progressive deterioration [9].

The phenotype is varied, and at least seven clinical subtypes of ALD have been described: childhood cerebral ALD, adolescent cerebral ALD, adult cerebral ALD, adrenomyeloneuropathy, asymptomatic and heterozygous women. These subtypes may be present within the same kinship [10]. Childhood cerebral ALD is the most common phenotype, with neurological impairment that can lead to death within a few months after the onset of symptoms, show-up usually between 4 and 8 years old. Adrenomyeloneuropathy is the mildest variant, mainly involving the spinal cord and peripheral nerves, with an average age of onset of approximately 7 years and a course that may extend for decades [11].

ALD is characterized by progressive neurological symptoms and dysfunction of the adrenal glands and testicles [12]. Virtually all men with ALD develop adrenal insufficiency and myelopathy [10].

The mode of inheritance of ALD is recessive linked to the $\mathrm{X}$ chromosome, so the possibility of a carrier's child developing ALD is $50 \%$, while $50 \%$ of nondescendent women will also be heterozygous carriers. All female descendants of affected women will be carriers [3].

\subsection{Clinical Manifestations}

ALD is a neurodegenerative disease in which all patients are born asymptomatic. The first manifestation of the disease in male patients is usually adrenal insufficiency, typically seen in childhood. Within adulthood, signs of myelopathy invariably develop [13], but progressive brain demyelination can occur in both childhood and adulthood, either as the first manifestation of the disease or after adrenal insufficiency [4].

Adrenomyeloneuropathy (AMN), the milder variant, most commonly manifests itself in an individual in the average age of twenty years with progressive stiffness and weakness of the legs, sphincter disorders, sexual dysfunction, and often impaired adrenocortical function; all symptoms are progressive over decades [14].

The brain form in childhood most commonly manifests between four and eight years of age. It initially resembles attention deficit hyperactivity disorder. Progressive impairment of cognition, behavior, vision, hearing, and motor func- 
tion follows initial symptoms and usually leads to total disability within six months to two years. Most individuals have impaired adrenocortical function at the time neurological disorders are first noticed [4].

Adult presentation is similar to childhood ALD and may initially appear as psychiatric disorders that resemble manifestations of obsessive-compulsive personality disorder. These psychiatric symptoms may precede overt motor or cognitive changes in a few years. Infections or head trauma may trigger the onset of the disease, but generally no extrinsic factors are identified [15]. Once the disease becomes inflammatory, it usually progresses rapidly to a devastating form, leading to a vegetative state within two to five years [16].

The variant called "Addison only" presents with primary adrenocortical insufficiency between 2 years and adulthood, most commonly at 7 years, with no evidence of neurological abnormality; however, some degree of neurological disability usually develops during the course of the disease [17].

\subsection{Etiopathogenesis}

ALD is caused by a mutation in the ABCD1 gene, which is located at Xq28 and contains 10 exons. It encodes a 745 amino acid transmembrane peroxisomic protein, called Adrenoleukodystrophy Protein (ALDP), a member of the ATPbinding cassette transport protein $(\mathrm{ABC})$ that family is responsible for the active transport of the enzyme acetylcoasynthetase that acts on $\beta$-oxidation of very long chain fatty acids (AGCML). Its mutation promotes VLCFA accumulation [18]. More than 1000 mutations in the ABCD1 gene have been identified and described [19].

Abnormal accumulation of AGCML can directly alter the adrenal gland's cellular function by altering the viscosity of the adrenocortical cell membrane, where the $\mathrm{ABCD} 1$ gene product normally resides, inhibiting the stimulatory effects of adrenocorticotropin (ACTH) on adrenocortical cells [20]. These data support the thesis that a decrease in trophic hormone responsiveness in ALD and AMN is secondary to changes in target tissue membrane microviscity and suggests a mechanism by which adrenal and gonadal insufficiency occurs in these patients. They could be responsible for at least part of the pathophysiology of ALD and AMN [21].

Adrenocortical cells in ALD patients, particularly those of the internal fascicular-reticular zone, become swollen and striated because of the accumulation of lamellae, lamellar-lipid profiles, and thin lipid fissures. Histochemical and biochemical studies suggest that these coverslips consist of esterified cholesterol molecules with saturated VLCFA. Striated material appears to lead to atrophy, cell dysfunction, and cell death. Ultimately, primary adrenal cortex atrophy occurs as a result of ACTH deficient action on adrenocortical cells [22].

\subsection{Adrenal Insufficiency}

The most frequent cause of adrenal insufficiency is autoimmune adrenalitis in developed countries. In developing countries, on the other hand, tuberculosis 
probably remains the most common cause of adrenal insufficiency. In young men, the presence of adrenal insufficiency should raise the possibility of adrenoleukodystrophy or even associated adrenomyeloneuropathy [23].

Primary adrenal insufficiency is often associated with AMN or ALD. This may be the only clinical expression of ALD ( $6 \%$ - $8 \%$ of cases). However, this may represent a bias because at least half of Addison cases are detected in ALD families where an index case has already been diagnosed. The exact prevalence of ALD among Addison patients is, however, unknown [24].

Patients with chronic adrenal insufficiency will experience fatigue, anorexia, nausea, vomiting, loss of appetite, weight gain or loss, and abdominal symptoms. Increased skin pigmentation may also be noted as a result of elevations of melanocyte stimulating hormone, a byproduct of ACTH. Common areas for observing hyperpigmentation include palmar folds, armpits, genitalia, areolas, scars, gums, and posterior region of helix to ear [25].

In an acute adrenal crisis, patients may have hypotension, hypoglycemia, altered mental status, and chronic symptoms already described. Sodium and potassium electrolyte changes (hyponatremia and hyperkalemia) are less common in ALD, but patients are described as having aldosterone response impairment to ACTH stimulation and require mineralocorticoid replacement [7].

VLCFA accumulation in adrenocortical cells appears to directly affect adrenal function. In the adrenal cortex, there is a preferential accumulation of VLCFA in the reticular zone and fasciculated zone, with relative preservation of the glomerular zone. As a result, adrenal manifestations of ALD tend to be more primary cortisol insufficiency as well as androgen deficiency (cortisol is mainly produced in the fasciculated zone and androgens are mainly produced in the reticular zone). VLCFAs can also be incorporated into lipid cell membranes and interfere with ACTH and gonadotropins that bind to their receptors. It has also been suggested that high levels of VLCFA lead to a relative scarcity of precursor cholesterol for cortisol and androgen steroidogenesis [19].

Primary adrenal insufficiency is a prominent feature of ALD and is characterized by high levels of adrenocorticotropic hormone (ACTH) and low cortisol levels. Currently, endocrine tests cannot distinguish whether adrenal insufficiency is due to ALD or other causes, such as those associated with an autoimmune response, which is the most prevalent, especially in adults [4].

\subsection{Diagnosis}

\section{Adrenal Insufficiency}

Initial outpatient testing should be a measurement of serum cortisol between 6 8 am and plasma ACTH. This approach exploits the fact that the cortisol peak occurs early in the morning [26]. This test is useful if the serum cortisol level is above $15 \mu \mathrm{g} / \mathrm{dL}$ (normal adrenal function) or below $3 \mu \mathrm{g} / \mathrm{dL}$ (indicates insufficient In the latter case, the plasma ACTH level will distinguish between primary and secondary adrenal insufficiency (AI) [26]. In primary AI, the ACTH level is almost invariably greater than $100 \mathrm{pg} / \mathrm{mL}$, whereas in secondary AI, plasma ACTH 
may be low or inappropriately normal [27].

If an outpatient has a morning serum cortisol between 3.1 and $14.9 \mu \mathrm{g} / \mathrm{dL}$, a dynamic adrenal function test is essential [28].

\subsection{CRH Stimulation Test}

$\mathrm{CRH}$ stimulation testing is based on the inability of the diseased adrenal gland to respond acutely to the injection of ACTH secreting cortisol. In the conventional, $250 \mu \mathrm{g}$ of synthetic ACTH is injected intravenously (or intramuscularly) and serum cortisol levels are measured 30 and 60 minutes later. Serum cortisol level greater than $18 \mu \mathrm{g} / \mathrm{dL}$ at any time is a normal response, regardless of baseline and time of day. Although some healthy individuals may peak below this cutoff level, a peak of less than $15 \mu \mathrm{g} / \mathrm{dL}$ is invariably abnormal [26].

Endocrine Society guidelines suggest that corticotropin stimulation testing is the best diagnostic test for identifying individuals with primary adrenal insufficiency regardless of age [29].

\subsection{Insulin Tolerance Test}

The insulin tolerance test measures the responsiveness of the patient's cortisol to intravenous insulin-induced hypoglycemia. This test is often considered the criterion standard because it assesses the ability of the entire hypothalamus-pituitary-adrenal axis (HHA) to respond to hypoglycemia. Following insulin administration (0.1 $\mathrm{IU} / \mathrm{kg}$ ), blood is collected during symptomatic hypoglycaemia (blood glucose should be below $40 \mathrm{mg} / \mathrm{dL}$ ). In obese patients with insulin resistance, the usual insulin dose should be increased to $0.15 \mathrm{IU} / \mathrm{kg}$. A serum cortisol peak greater than $18 \mu \mathrm{g} / \mathrm{dL}$ is considered normal. This test has the advantage that it can also assess growth hormone reserve in patients with pituitary or hypothalamic disease. It is contraindicated in patients older than 60 years and in those with a history of seizures or with or suspected coronary artery disease. In addition, it requires strict medical supervision and trained personnel [26].

Once the diagnosis of adrenal insufficiency is established, the physician should perform a careful neurological examination, keeping in mind that AI may appear long before neurological symptoms. Several known infectious processes may be associated with AIDS, cytomegalovirus, tuberculosis, cryptococcosis, toxoplasmosis, atypical microbacteriosis and histoplasmosis. All of these causes can lead to destruction of the adrenal gland. Among the medications, ketoconazole and etomidate are the cause of AI [26].

\subsection{VLCFA Dosage}

VLCFA concentration analysis is the test used for confirmatory diagnosis of $\mathrm{X}$-ALD. This is based on the significant increase in hexacosanoic acid (C26:0), the ratios of hexacosanoic and tetracosanoic acids (C26:0/C24:0) and between hexacosanoic and docosanoic acids (C22:0) [5].

Once ALD is confirmed or if patients have neurological symptoms, patients 
should be referred immediately to a neurologist for formal and neuroimaging evaluation [19].

\subsection{Molecular Analysis of the ABCD1 Gene}

When ALDP is positive, molecular analysis of the ABCD1 gene is suggested, determining the mutation responsible for the development of the disease. This medium is presented as a support for genetic follow-up [30].

\subsection{Prenatal Diagnosis}

Prenatal diagnosis is made by detecting ALD protein (ALDP) and AGCML in chorionic villus and amniotic fluid, since the chorionic villus cells present great expression of this protein and the fats in question [12] [30]. Detection of ALDP is performed by immunofluorescence and/or immunoblotting, made possible by the availability of antibodies formed against different sites of this same protein [5].

\subsection{Imaging Exam}

Magnetic resonance imaging (MRI) is a very important tool in ALD diagnosis, since it detects cerebral involvement of the white matter and allows the distinction of gliosis regions. Imaging on MRI is variable, but in typical presentation it begins in the corpus callosum and adjacent white matter in the form of three layers. In the center there is an area of hyposignal necrosis in T1-weighted sequences and hypersignal in $\mathrm{T} 2$-weighted sequences, bordered by an intermediate demyelinating area with perivascular inflammation that is responsible for the breakdown and marginal enhancement by gadolinium, usually bilateral and bevelled with foci of discontinuity near the adjacent cortex. Finally, around the enhancement area is another non-inflammatory demyelination zone and edema [9].

The gold standard for assessing disease progression was proposed by Loes in 1994 on a magnetic resonance severity scale with scores from 0 to 34 points using a scoring system based on the location and extent of involvement and assessment of the presence of local atrophy or global. The locations selected for the severity score are the parietoccipital, anterior and frontal temporal white matter, corpus callosum, visual and auditory pathways, projection fibers, cerebellum and base nuclei. The severity score assists in determining the extent of brain myelin damage. A very early stage is considered if scores are less than 4 , an early stage is considered with scores between 4 and 8, a late stage is between 9 and 13 and a very late stage if scores are greater than 13 . The Loes's score is one of the parameters used in follow-up and guides therapeutic decisions for hematopoietic cell transplantation [31].

This severity scale based on the MRI assessment was designed specifically for adrenoleukodystrophy and was shown to correlate with the severity of neurological deficits and to be predictive of disease progression. Different brain regions are considered in the Loes score. Each area is rated to the 0 if normal; 0.5 if 
the involvement is unilateral; and 1 if the lesion or atrophy is bilateral. The maximum severity score is 34 ; a score of 1 is considered abnormal [32].

\subsection{Treatment of Adrenal Insufficiency}

For patients with adrenal insufficiency, replacement therapy saves lives. Glucocorticoids (20 to $30 \mathrm{mg}$ hydrocortisone or 25 to $50 \mathrm{mg}$ cortisone acetate) are required in two or three daily doses. The first dose is given in the morning, the second in the early afternoon (about 6 to 8 hours after the first) and the third in the early evening. A 24 -hour free cortisone urine test is considered a good way to assess the adequacy of cortisone intake [29]. In Brazil, as there is no commercial availability of oral hydrocortisone, in situations of difficulty in handling this drug, it can be used prednisone at a dose of $5-10 \mathrm{mg} /$ day in single or fractionated doses [33].

A single dose of 0.05 to $0.1 \mathrm{mg}$ fludrocortisone is usually sufficient for mineralocorticoid replacement [26]. The response to mineralocorticoid replacement is clinically assessed by assessing salt desire or faintness, the presence of orthostatic hypotension and the presence of peripheral edema, although it is of low sensitivity. Overall well-being, normal-range electrolytes and normal blood pressure with no evidence of postural hypotension indicate adequate mineralocorticoid replacement. In addition, plasma renin activity in the upper reference range has been found to be a useful marker for a correct dose of mineralocorticoid [29].

Patients should also receive a high sodium diet. Measuring blood pressure, serum electrolytes, and plasma renin activity levels are useful for optimizing therapy [34]. Patients should have a medical identification card describing their current therapy and guidelines for dealing with emergency situations such as illness, fever, injuries, vomiting, surgical procedures, tooth extractions or pregnancy. In such situations, glucocorticoid intake needs to be doubled or tripled, possibly by intramuscular or intravenous injection [27].

Corticosteroid and mineralocorticoid replacement therapies should suppress excessive CRH secretion, ACTH, and resting renin levels. Normal daily cortisol production has been $6-7 \mathrm{mg} / \mathrm{m}^{2} /$ day in children and adolescents. This rate translates to about $10-12 \mathrm{mg} / \mathrm{m}^{2} /$ day oral hydrocortisone, to allow loss of absorption reduction, hepatic metabolism and metabolic bioavailability. In children, the preferred cortisol replacement is hydrocortisone at doses of $10-20$ $\mathrm{mg} / \mathrm{m}^{2} /$ day in three doses [26].

\subsection{Bone Marrow Transplant}

Allogeneic bone marrow transplantation is the only treatment with proven efficacy in cerebral ALD. It disrupts disease progression and improves survival in presymptomatic patients [10]. Most cerebral ALD patients die within a decade of being diagnosed. Among symptomatic patients with a high burden of brain disease at diagnosis, transplant outcomes are often unfavorable [6]. 
The mechanism that makes hematopoietic stem cell transplantation effective is not yet known, but it is believed that the lack of effect of myeloablative conditioning raises the possibility of replacing activated microglia (macrophages, lymphocytes) with normal microglia, which would be sufficient to disrupt the cerebral inflammatory process [35].

Due to concerns about high morbidity and mortality from bone marrow transplantation, a common and serious complication in allogeneic modality, only patients with ALD with Loes scores greater than 1 and less than 9, as well as intelligence quotients greater than 80, are considered candidates for hematopoietic stem cell transplantation [36].

Therefore bone marrow transplantation has been shown to be effective if performed at an early stage of neurodegeneration [36]. Thus, rapid identification of potential matched hematopoietic stem cell donors is important to ensure successful treatment and improve survival of these patients [6].

\subsection{Gene Therapy}

Correction of metabolic defect in cultured cells of ALD patients has been demonstrated following the addition of ALDP cDNA via retroviral systems and found to normalize the peroxisomal $\beta$-oxidation activity of VLCFA in skin fibroblasts and in hematopoietic cells. The use of this type of vector may be useful if applied to autologous bone marrow transplantation in the treatment of ALD [37].

\subsection{Lorenzo's Oil}

Diets low in VLCFA and supplemented with Lorenzo oil (a mixture of erunic and oleic acid) have been suggested to stabilize the disease in mildly affected or asymptomatic patients. Lorenzo's oil reduces the synthesis of VLCFA by competitively inhibiting the enzymes responsible for elongating saturated fatty acids, because the enzyme responsible for metabolizing saturated fats is the same as degrading monounsaturated fats. Lovastatin and sodium phenylacetate normalize the level of LCFA and may be possible therapeutic agents [38]. Lorenzo oil is composed of 4 parts of oleic acid and 1 part of erucic acid. It is given according to the patient's age, body weight and health conditions at a dose of 2 - 3 $\mathrm{ml} / \mathrm{kg} /$ day in 2 to 3 daily doses, and may be taken in pure or in combination with other low-fat foods [5].

The use of Lorenzo's oil by neurologically asymptomatic children slows the development of brain white matter degeneration and adrenal gland lesions [39]. However Lorenzo's oil has shown little therapeutic benefit [40].

\section{Conclusions}

ALD leads to a pathognomonic accumulation of very long chain fatty acids in the brain's white matter, adrenal gland, fibroblasts, and plasma caused by impaired oxidation of these fatty acids in peroxisomes. The metabolic defect is 
most severely expressed in the brain, adrenal cortex and testes. The reason why these tissues are particularly susceptible to VLCFA accumulation remains unclear.

Because of the potential serious consequences of undiagnosed adrenal insufficiency, start adrenal screening as soon as the diagnosis of ALD is recommended. Treatment of adrenal insufficiency is mandatory and life-saving, but unfortunately, does not change the course of neurological symptoms.

\section{Conflicts of Interest}

The authors declare no conflicts of interest regarding the publication of this paper.

\section{References}

[1] Bezman, L., et al. (2001) Adrenoleukodystrophy: Incidence, New Mutation Rate, and Results of Extended Family Screening. Annals of Neurology, 49, 512-517. https://doi.org/10.1002/ana.101

[2] Kemp, S., et al. (2016) Adrenoleukodystrophy-Neuroendocrine Pathogenesis and Redefinition of Natural History. Nature Reviews Endocrinology, 12, 606-615. https://doi.org/10.1038/nrendo.2016.90

[3] Pereira Fdos, S., et al. (2012) Mutations, Clinical Findings and Survival Estimates in South American Patients with X-Linked Adrenoleukodystrophy. PLoS ONE, 7, e34195. https://doi.org/10.1371/journal.pone.0034195

[4] Engelen, M.S. and Kemp, B.T. (2014) X-Linked Adrenoleukodystrophy: Pathogenesis and Treatment. Current Neurology and Neuroscience Reports, 14, 486. https://doi.org/10.1007/s11910-014-0486-0

[5] Maciel, K.A.C. and Oliveira, M.S. (2012) Diagnóstico e tratamento da adrenoleucodistrofia ligada ao X. XII Safety, Health and Environment World Congress, São Paulo, 22-25 July 2012, 10.

[6] Eichler, F., et al. (2017) Hematopoietic Stem Cell Gene Therapy for Cerebral Adrenoleukodystrophy. The New England Journal of Medicine, 377, 1630-1638.

[7] Kaltsas, G., Kanakis, G. and Moser, H. (2015) Adrenal Insufficiency Due to X-Linked Adrenoleukodystrophy. MDText.com, South Dartmouth.

[8] Moser, H., Dubey, P. and Fatemi, A. (2004) Progress in X-Linked Adrenoleukodystrophy. Current Opinion in Neurology, 17, 263-269.

https://doi.org/10.1097/00019052-200406000-00005

[9] Santos, A.C. (2014) Adrenoleucodistrofia ligada ao X: Diagnóstico e quantificação da progressão. Radiologia Brasileira, 47, 7-8. https://doi.org/10.1590/0100-3984.2014.47.6e2

[10] Engelen, M.S. (2017) Optimizing Treatment for Cerebral Adrenoleukodystrophy in the Era of Gene Therapy. The New England Journal of Medicine, 377, 1682-1684. https://doi.org/10.1056/NEJMe1709253

[11] Jorge, P., et al. (1994) X-Linked Adrenoleukodystrophy in Patients with Idiopathic Addison Disease. European Journal of Pediatrics, 153, 594-597. https://doi.org/10.1007/BF02190668

[12] Spurek, M., Taylor-Gjevre, R., Van Uum, S. and Khandwala, H.M. (2004) Adrenomyeloneuropathy as a Cause of Primary Adrenal Insufficiency and Spastic Paraparesis. Canadian Medical Association Journal, 171, 1073-1077. 
https://doi.org/10.1503/cmaj.1032006

[13] Aubourg, P. (2007) X-Linked Adrenoleukodystrophy. Annales d Endocrinologie, 68, 403-411.

[14] Melhem, E.R., et al. (2000) X-Linked Adrenoleukodystrophy: The Role of Contrast-Enhanced MR Imaging in Predicting Disease Progression. American Journal of Neuroradiology, 21, 839-844.

[15] Moser, H.W., et al. (2000) X-Linked Adrenoleukodystrophy: Overview and Prognosis as a Function of Age and Brain Magnetic Resonance Imaging Abnormality. A Study Involving 372 Patients. Neuropediatrics, 31, 227-239. https://doi.org/10.1055/s-2000-9236

[16] Salsano, E., et al. (2014) Brain Fluorodeoxyglucose PET in Adrenoleukodystrophy. Neurology, 83, 981-989. https://doi.org/10.1212/WNL.0000000000000770

[17] Raymond, G.V., Moser, A.B. and Fatemi, A. (2018) X-Linked Adrenoleukodystrophy. Gene Reviews.

[18] Higgins, C.F. (1992) ABC Transporters: From Microorganisms to Man. Annual Review of Cell Biology, 8, 67-113. https://doi.org/10.1146/annurev.cb.08.110192.000435

[19] Burtman, E. and Regelmann, M.O. (2016) Endocrine Dysfunction in X-Linked Adrenoleukodystrophy. Endocrinology and Metabolism Clinics of North America, 45, 295-309. https://doi.org/10.1016/j.ecl.2016.01.003

[20] Whitcomb, R.W., Linehan, W.M. and Knazek, R.A. (1988) Effects of Long-Chain, Saturated Fatty Acids on Membrane Microviscosity and Adrenocorticotropin Responsiveness of Human Adrenocortical Cells in Vitro. Journal of Clinical Investigation, 81, 185-188. https://doi.org/10.1172/JCI113292

[21] Knazek, R.A., Rizzo, W.B., Schulman, J.D. and Dave, J.R. (1983) Membrane Microviscosity Is Increased in the Erythrocytes of Patients with Adrenoleukodystrophy and Adrenomyeloneuropathy. Journal of Clinical Investigation, 72, 245-248. https://doi.org/10.1172/JCI110963

[22] Powers, J.M. and Schaumburg, H.H. (1973) The Adrenal Cortex in Adrenoleukodystrophy. Archives of Pathology \& Laboratory Medicine, 96, 305-310.

[23] Hannah-Shmouni, F., et al. (2016) Cortisol in the Evaluation of Adrenal Insufficiency. JAMA, 316, 535-536. https://doi.org/10.1001/jama.2016.8360

[24] Laureti, E., et al. (1996) X-Linked Adrenoleukodystrophy Is a Frequent Cause of Idiopathic Addison's Disease in Young Adult Male Patients. Journal of Clinical Endocrinology and Metabolism, 81, 470-474. https://doi.org/10.1210/jc.81.2.470

[25] Shulman, D.I., et al. (2007) Adrenal Insufficiency: Still a Cause of Morbidity and Death in Childhood. Pediatrics, 119, e484-e494.

https://doi.org/10.1542/peds.2006-1612

[26] Salvatori, R. (2005) Adrenal Insufficiency. JAMA, 294, 2481-2488. https://doi.org/10.1001/jama.294.19.2481

[27] Oelkers, W. (1996) Adrenal Insufficiency. The New England Journal of Medicine, 335, 1206-1212. https://doi.org/10.1056/NEJM199610173351607

[28] Fleseriu, M., Hashim, I.A., Karavitaki, N., Melmed, S., Murad, M.H., Salvatori, R. and Samuels, M.H. (2016) Hormonal Replacement in Hypopituitarism in Adults: An Endocrine Society Clinical Practice Guideline. The Journal of Clinical Endocrinology \& Metabolism, 101, 3888-3921. https://doi.org/10.1210/jc.2016-2118

[29] Bornstein, S.R., et al. (2016) Diagnosis and Treatment of Primary Adrenal Insufficiency: An Endocrine Society Clinical Practice Guideline. The Journal of Clinical 
Endocrinology \& Metabolism, 101, 364-389. https://doi.org/10.1210/jc.2015-1710

[30] Wanders, R.J.A., Mooyer, P.W., Dekker, C. and Vreken, P. (1998) X-Linked Adrenoleukodystrophy: Improved Prenatal Diagnosis Using Both Biochemical and Immunological Methods. Journal Inherited Metabolic Disease, 21, 285-287. https://doi.org/10.1023/A:1005336726127

[31] Ono, S.E., et al. (2014) X-Linked Adrenoleukodystrophy: Correlation between Loes Score and Diffusion Tensor Imaging Parameters. Radiologia Brasileira, 47, 342-349. https://doi.org/10.1590/0100-3984.2013.1886

[32] Loes, D.J., et al. (2003) Analysis of MRI Patterns Aids Prediction of Progression in X-Linked Adrenoleukodystrophy. Neurology, 61, 369-374. https://doi.org/10.1212/01.WNL.0000079050.91337.83

[33] Silva, R.C., et al. (2004) Insuficiência Adrenal Primária no Adulto: 150 Anos Depois de Addison. Arquivos Brasileiros de Endocrinologia \& Metabologia, 48, 724-738. https://doi.org/10.1590/S0004-27302004000500019

[34] Betterle, C. and Morlin, L. (2011) Autoimmune Addison's Disease. Endocrine Development, 20, 161-172. https://doi.org/10.1159/000321239

[35] Ferrer, I., Aubourg, P. and Pujol, A. (2010) General Aspects and Neuropathology of X-Linked Adrenoleukodystrophy. Brain Pathology, 20, 817-830. https://doi.org/10.1111/j.1750-3639.2010.00390.x

[36] Vogel, B.H., et al. (2015) Newborn Screening for X-Linked Adrenoleukodystrophy in New York State: Diagnostic Protocol, Surveillance Protocol and Treatment Guidelines. Molecular Genetics and Metabolism, 114, 599-603. https://doi.org/10.1016/j.ymgme.2015.02.002

[37] Jorge, P.M.V. (2000) Adrenoleucodistrofia ligada ao cromossoma X: Estudos bioquímicos e moleculares. Porto, Tese (Doutor em Ciências Biomédicas, especialidade Genética Humana) Universidade do Porto, 153 p.

[38] Cartier, N., et al. (2009) Hematopoietic Stem Cell Gene Therapy with a Lentiviral Vector in X-Linked Adrenoleukodystrophy. Science, 326, 818-823. https://doi.org/10.1126/science.1171242

[39] Vargas, C.R., et al. (2000) X-Linked Adrenoleukodystrophy: Clinical and Laboratory Findings in 15 Brazilian Patients. Genetics and Molecular Biology, 23, 261-264. https://doi.org/10.1590/S1415-47572000000200002

[40] Ten, S., et al. (2001) Addison's Disease. The Journal of Clinical Endocrinology \& Metabolism, 86, 2909-2922. https://doi.org/10.1210/jc.86.7.2909 